\title{
Organization of the biosynthetic gene cluster in Streptomyces sp. DSM 4137 for the novel neuroprotectant polyketide meridamycin
}

\author{
Yuhui Sun, Hui Hong, Markiyan Samboskyy, Tatiana Mironenko, \\ Peter F. Leadlay and Stephen F. Haydock \\ Department of Biochemistry, University of Cambridge, 80 Tennis Court Road, Cambridge \\ CB2 1GA, UK
}

Correspondence

Stephen F. Haydock

stephen.haydock@

addenbrookes.nhs.uk

Received 30 May 2006

Revised 8 August 2006

Accepted 29 August 2006

\begin{abstract}
Meridamycin is a non-immunosuppressant, FKBP-binding macrocyclic polyketide, which has major potential as a neuroprotectant in a range of neurodegenerative disorders including dementia, Parkinson's disease and ischaemic stroke. A biosynthetic cluster predicted to encode biosynthesis of meridamycin was cloned from the prolific secondary-metabolite-producing strain Streptomyces sp. DSM 4137, not previously known to produce this compound, and specific gene deletion was used to confirm the role of this cluster in the biosynthesis of meridamycin. The meridamycin modular polyketide synthase consists of 14 extension modules distributed between three giant multienzyme proteins. The terminal module is flanked by a highly unusual cytochrome P450-like domain. The characterization of the meridamycin biosynthetic locus in this readily manipulated streptomycete species opens the way to the engineering of new, altered meridamycins of potential therapeutic importance.
\end{abstract}

\section{INTRODUCTION}

Polyketide macrolides constitute a large family of bioactive natural products and include many compounds with important clinical applications in both human and veterinary medicine. Meridamycin is a 27 -membered macrocyclic polyketide, isolated independently from two different streptomycete strains (Fehr et al., 1994; Salituro et al., 1995). Although it lacks immunosuppressant activity, it shares the structural motif common to the macrocyclic polyketide immunosuppressants rapamycin (Schwecke et al., 1995), FK506 (Motamedi \& Shafiee, 1998) and FK520 (Wu et al., 2000), which is known to interact with the hydrophobic cavity on the surface of FK506-binding protein-12 (FKBP-12) (Van Duyne et al., 1991, 1993). This structural moiety is proposed to mimic a natural peptide substrate and hence inhibit the peptidyl-prolyl isomerase activity of FKBP-12. Meridamycin, however, differs from rapamycin, FK520 and FK506 in the absence of the substituted cyclohexane ring characteristic of these molecules (Fig. 1). Meridamycin competitively inhibits the binding of FK506 and rapamycin to FKBP-12. It also inhibits the immunosuppressant action of FK506 in a

Abbreviations: AT, acyltransferase; FKBP, FK506-binding protein; LCMS, liquid chromatography-mass spectrometry; NRPS, non-ribosomal peptide synthetase; PKS, polyketide synthase.

The GenBank/EMBL/DDBJ accession number for the sequence data reported in this paper is $\mathrm{D0885223.}$ functional assay of interleukin (IL)-2 transcription; and that of rapamycin in an IL-6-dependent cell-proliferation assay.

Rapamycin (sirolimus), FK506 (tacrolimus) and more recently FK520 (pimecrolimus) have now become well established in clinical practice. In addition to immunosuppressant and anti-proliferative activity, they also possess neurotrophic activity through binding to neuroimmunophilin FKBPs that are markedly enriched in the central nervous system and in peripheral nerves. These agents can stimulate nerve growth regeneration in a range of animal models. They are currently under investigation for the treatment of a number of important human neurological disorders, including multiple sclerosis (Lubetski et al., 2005), traumatic spinal cord injury (Sosa et al., 2005) and Parkinson's disease (Tanaka \& Ogawa, 2004). It is clear that the immunosuppressant and neurotrophic properties of FKBP-binding macrolides represent separate and distinct pharmacological effects. Neuroregenerative activity, in the absence of immunosuppressant activity, is shown by compounds which bind to FKBP but do not interact with the effector proteins calcineurin or mTOR (Steiner et al., 1997). It has recently been proposed that the neurotrophic activity may in fact be due to binding to targets other than FKBP-12. In FKBP-12 knockout mice, FKBP-12-binding ligands retain their neurotrophic activity on hippocampal neurones (Gold et al., 1999) and FK506 is still able to protect dopaminergic neurones against $\mathrm{MPP}^{+}$-induced toxicity (Guo et al., 2001). Both studies indicate that binding to 


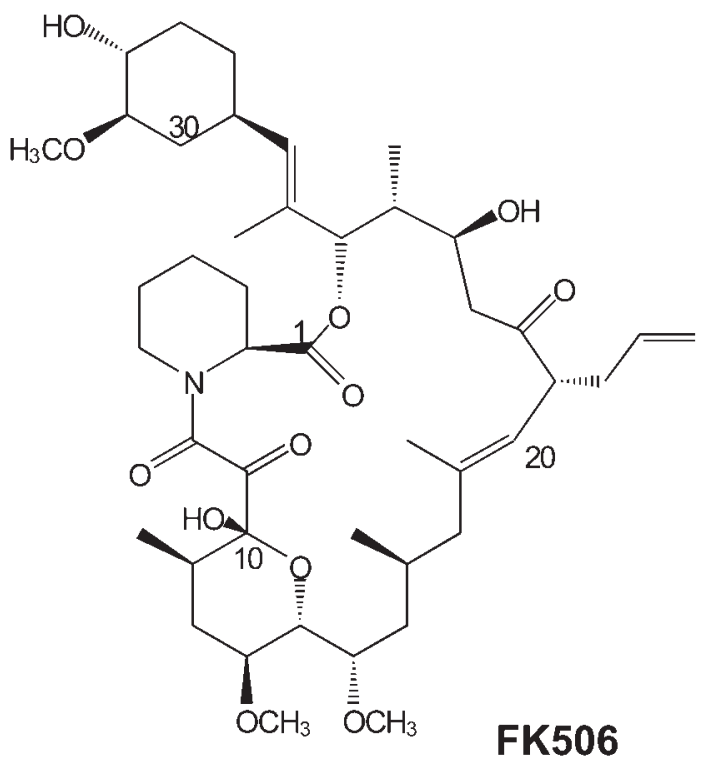

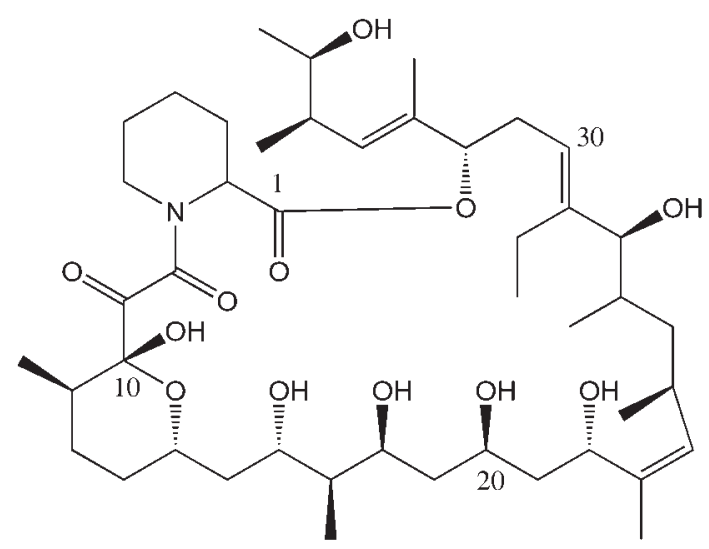

Meridamycin

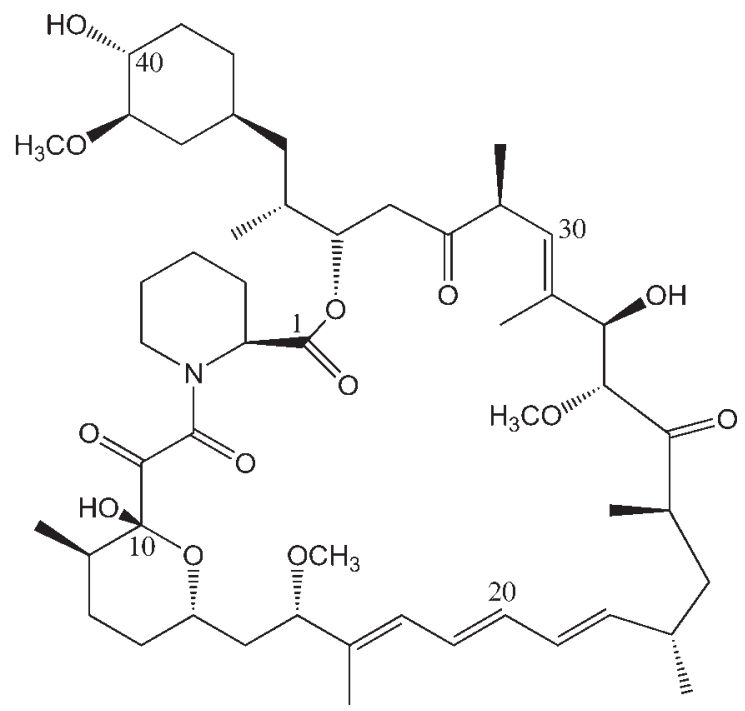

Rapamycin

Fig. 1. FKBP-binding ligands meridamycin, rapamycin, FK506 and FK520 show common structural features.

FKBP-12 is not required for neurotrophic activity. Similarly, non-immunosuppressant derivatives of FK506 that lack FKBP-12 binding are found to stimulate neurite elongation in SH-SY5Y cells (Gold et al., 2005). It is likely that binding to members of the FKBP family other than FKBP-12 underlies the neurotrophic activity of these agents. In particular, FKBP-52 has been proposed as the true target (Gold et al., 1999). In keeping with these observations, meridamycin has recently been shown to possess similar neuroprotectant and neurotrophic activities. The absence of immunosuppressant activity is a considerable advantage as such activity results in a range of serious and unwanted sideeffects. The production of modified meridamycin analogues by manipulation of the biosynthetic gene cluster would be an attractive approach to compounds of potential utility in a range of neurodegenerative disorders with huge unmet need, causing enormous morbidity and mortality.

Our analysis of the biosynthesis of meridamycin was prompted by our investigation of the remarkable polyketide biosynthetic potential of Streptomyces sp. DSM 4137. This organism contains biosynthetic gene clusters for at least 11 complex modular polyketides as well as numerous aromatic polyketides, non-ribosomally synthesized peptides and other secondary metabolites. We have completed an analysis of the gene clusters encoding the novel macrodiolide elaiophylin (Haydock et al., 2004) from this organism, and of the cluster encoding biosynthesis of the related polyethers nigericin and abierixin (B. M. Harvey and others, unpublished). Our analysis of the remaining clusters has 
now led to the identification of a $100 \mathrm{kbp}$ locus whose organization is entirely consistent with the biosynthesis of meridamycin. We have confirmed that the strain produces meridamycin, and have used targeted gene deletion to confirm that the cloned genes govern this pathway. The genetic manipulation of the biosynthetic genes for meridamycin biosynthesis in Streptomyces sp. DSM 4137 represents a convenient system for the generation of a family of meridamycin metabolites with potential in the treatment of a range of debilitating neurological disorders.

While the present paper was in preparation, a chromosomal DNA sequence reported to encode meridamycin biosynthesis in Streptomyces sp. NRRL 30748 was deposited in GenBank, under accession number DQ351275, to accompany a brief description and a number of claims relating to the sequence, recently published in US Provisional Patent Application 60/664,483, originally filed on March 23, 2005. Comparison of our sequence with that of the sequence from Streptomyces sp. NRRL 30748 has helped to confirm the boundaries of the cluster but has also identified significant and unexpected differences in gene organization between the two clusters. An account of the meridamycin cluster in Streptomyces sp. NRRL 30748 has recently been published (He et al., 2006).

\section{METHODS}

Bacterial strains and cloning vectors. Streptomyces sp. DSM 4137 was the kind gift of Dr Lake Ee Khaw, Department of Biochemistry, University of Cambridge. Streptomyces sp. DSM 7348 is a known meridamycin-producing strain and was the kind gift of Dr Rose Sheridan, Biotica Technology Ltd, Chesterford Research Park, Essex. The following Escherichia coli strains were used: DH10B (Gibco-BRL) for routine cloning; XL-1 Blue MR for cosmid library construction; and ET12567 (MacNeil et al., 1992), into which the helper plasmid pUZ8002 (Paget et al., 1999) was cloned, as a donor strain for conjugation into strain DSM 4137. Disruption of the meridamycin gene cluster was carried out using plasmid pYH24, derived from pJTU412 and pHZ1358 (Sun et al., 2002). Routine subcloning was performed in pSHG397 (Hashimoto-Gotoh et al., 1995).

Culture conditions and molecular genetic procedures. E. coli strains were grown on $2 \times$ TY medium supplemented with appropriate antibiotics. Streptomyces sp. DSM 4137 and Streptomyces sp. DSM 7348 were maintained on SFM medium (Kieser et al., 2000). Liquid cultures of DSM 4137 for isolation of genomic DNA were grown in tryptone soya broth (Difco). Cultures of strains DSM 4137, DSM 7348 and disruptants of DSM 4137 to be used in analysis of meridamycin production were grown in $50 \mathrm{ml}$ SFM liquid medium in a $250 \mathrm{ml}$ flask for 6 days at $30^{\circ} \mathrm{C}$. Standard genetic techniques for $E$. coli and for in vitro DNA manipulations were as described by Sambrook et al. (1989). Total DNA was isolated from DSM 4137 by 'procedure B' of Kieser et al. (2000). For the generation of the cosmid library, total DNA was partially digested with Sau3AI, dephosphorylated with shrimp alkaline phosphatase, ligated directly into pSuperCos and packaged with Gigapack Gold packaging extract (Stratagene) without size fractionation. All procedures were in accordance with the manufacturer's recommendations. Initial screening of the cosmid library of DSM 4137 was with a $9.5 \mathrm{kbp} \mathrm{KpnI} \mathrm{fragment} \mathrm{from} \mathrm{the} \mathrm{rapamycin} \mathrm{PKS} \mathrm{cluster} \mathrm{(Schwecke}$ et al., 1995) encoding ketosynthase (KS), acyltransferase (AT), dehydratase $(\mathrm{DH})$, ketoreductase $(\mathrm{KR})$ and acyl carrier protein (ACP) domains. Colony hybridization of the cosmid library was performed with a digoxigenin-labelled probe in accordance with the manufacturer's recommendations (Boehringer Mannheim). Cosmid DNA for complete sequencing was prepared using Qiagen midi-prep DNA purification kits from $50 \mathrm{ml}$ liquid cultures in $2 \times$ LB medium containing ampicillin and kanamycin. The complete sequence of each cosmid was obtained from overlapping Sau3AI fragments obtained after a partial digestion of the parent cosmid. The $2-5 \mathrm{kbp}$ fraction of this digest was eluted from an agarose gel using the Gene Clean kit (Bio 101, Bio-Rad) and subcloned into pSHG397 (HashimotoGotoh et al., 1995).

For construction of the plasmid used for disruption of the mer gene cluster, two DNA fragments, a $4164 \mathrm{bp} \mathrm{BclI-BamHI}$ fragment and a 4434 bp BclI fragment, were cloned from cosmids FB4 and H3G7 covering the mer gene cluster, respectively, then inserted into the unique BamHI restriction site of the delivery vector pYH7 with its natural orientation to give the plasmid pYH24. The plasmid pYH24 contains an origin of replication and can replicate in Streptomyces sp. DSM 4137.

The following primers were used for screening double crossover mutants in this study: mer-3, 5' -AAAAGTATGGGTTGACAGCG-3'; mer-4, 5' -CGGCACGATGCGACA-3'. Genomic PCR with the mer-3 and mer- 4 primers was performed in a final volume of $30 \mu \mathrm{l}$ for 30 cycles of amplification using a programmer $\left(94{ }^{\circ} \mathrm{C}, 5 \mathrm{~min} ; 94^{\circ} \mathrm{C}, 30 \mathrm{~s}\right.$; $\left.55^{\circ} \mathrm{C}, 40 \mathrm{~s} ; 72^{\circ} \mathrm{C}, 50 \mathrm{~s} ; 72{ }^{\circ} \mathrm{C}, 5 \mathrm{~min}\right)$. A PCR product (894 bp) was recovered from gel for sequencing. Southern hybridization was carried out with digoxigenin-labelled probes. Sequencing of DNA fragments generated by PCR amplification was performed directly using automated DNA sequencing as described below.

Extraction, isolation and characterization of meridamycin. Broths derived from $50 \mathrm{ml}$ cultures of DSM 7348 (an authentic meridamycin producer), DSM 4137 and disruptants were extracted with equal volumes of ethyl acetate. The residue, after evaporation of the solvent, was dissolved in $0.8 \mathrm{ml}$ methanol and subjected to liquid chromatography-mass spectrometry (LC-MS) analysis. On-line LCMS and LC-MS/MS analysis were carried out on a LCQ mass spectrometer (Thermo Finnigan) using positive electrospray ionization. The LCQ was coupled with an HP 100 LC (Agilent) fitted with a Prodigy C18 column $(5 \mu, 4 \cdot 6 \times 250 \mathrm{~mm})$ (Phenomenex). Samples were eluted with a 40 min gradient from 25 to $100 \%$ acetonitrile in water. High-resolution mass analysis was performed on a BioApex II (4.7 Tesla) FTICR mass spectrometer (Bruker Daltonics).

DNA sequencing analysis. Automated DNA sequencing was carried out on double-stranded DNA templates by the dideoxynucleotide chain-termination method in the University of Cambridge, Department of Biochemistry DNA sequencing facility. An Applied Biosystems 800 molecular biology CATALYST robot was used to apply Taq dideoxy terminator sequencing reactions (Big Dye Terminator kit, $\mathrm{ABI}$ ) to an $\mathrm{ABI} 373 \mathrm{~A}$ sequencer according to the manufacturer's protocols. Cosmid DNA for initial end sequencing was prepared from overnight growth of $1 \mathrm{ml}$ cultures in 96-well plates in $2 \times$ LB medium containing kanamycin and ampicillin. The complete sequence of the cosmid was obtained from analysis of overlapping Sau3AI fragments obtained via a partial digestion of the parent cosmid. The $2-5 \mathrm{kbp}$ size fraction of this digest was eluted from an agarose gel using the Gene Clean kit (Bio 101, Bio-Rad) and subcloned into the BamHI site of dephosphorylated pSHG397 (Hashimoto-Gotoh et al., 1995). These random subclones were sequenced using template DNA obtained from $1 \mathrm{ml}$ cultures grown overnight at $37^{\circ} \mathrm{C}$ in 96-well plates, using the universal forward and reverse sequencing primers. SeqEd version 1.03 was used for sequence editing. Database searches used the BLAST algorithm. Sequence assembly employed the GAP (Genome Assembly Program) version 4.2 (Bonfield et al., 1995). To fill remaining contig gaps and 
resolve any remaining ambiguities, custom-designed oligonucleotide primers were used to sequence from selected subclones or from the parent cosmid. Both strands were completely sequenced with a mean eightfold coverage per base.

\section{RESULTS AND DISCUSSION}

\section{Cloning and sequencing of the meridamycin biosynthetic cluster}

A genomic library of strain DSM 4137 was constructed in pSuperCos vector (Stratagene). This was screened by hybridization with a $9.5 \mathrm{kbp} K p n \mathrm{I}$ DNA fragment of rapA of the rapamycin PKS of Streptomyces hygroscopicus as described previously (Haydock et al., 2004). It was concluded on the basis of stringent hybridization conditions that $8 \%$ of the genome of this organism encoded type I PKS genes. To date, we have identified 11 type I PKS gene clusters in the genome of this organism. We are in the process of characterizing all of these loci. We have already reported the complete sequence of the cluster responsible for biosynthesis of the macrodiolide elaiophylin from this prolific organism (Haydock et al., 2004). Analysis of the DNA sequence of four overlapping cosmid clones that comprised another of these loci led to the identification of a cluster predicted to encode the biosynthesis of the macrolide meridamycin. This locus spans approximately $95 \mathrm{kbp}$. Extensive sequencing of flanking regions revealed the likely boundaries of the gene cluster.

\section{Confirmation of the identity of the meridamycin biosynthetic locus by gene disruption}

LC-MS analysis of the extracts of DSM 4137 showed a peak at $27 \cdot 04 \mathrm{~min}$ with $[\mathrm{M}+\mathrm{Na}]^{+}$at $844 \cdot 5 \mathrm{~m} / \mathrm{z}$ (Fig. 2a). High-

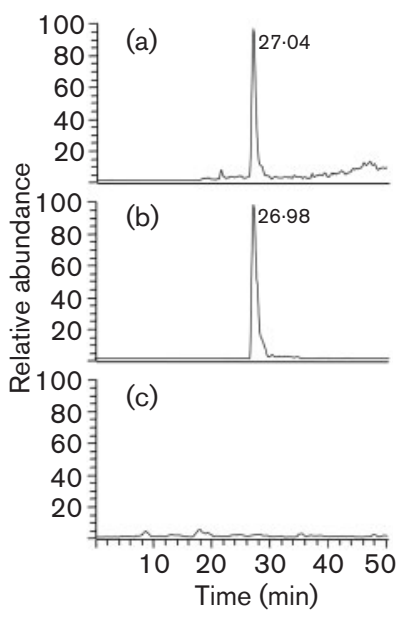

Fig. 2. LC-MS analysis of meridamycin $\left([\mathrm{M}+\mathrm{Na}]^{+}:\right.$844.5) production from cultural broth extracts. (a) lon trace of $\mathrm{m} / \mathrm{z}$ : 844.5 from DSM 4137 (wild-type); (b) ion trace of $\mathrm{m} / \mathrm{z}$ : 844.5 from the meridamycin-producing strain DSM 7348; (c) ion trace of

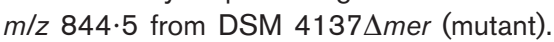

resolution mass analysis confirmed its formula as $\mathrm{C}_{45} \mathrm{H}_{75} \mathrm{NO}_{12} \mathrm{Na}^{+}$(Obs. 844.5187, Cal. 844.5181). To further confirm the production of meridamycin from strain DSM 4137, the meridamycin-producing strain Streptomyces sp. DSM 7348 was grown, harvested and analysed under the same conditions as for DSM 4137. LCMS analysis of this strain clearly showed that meridamycin with $[\mathrm{M}+\mathrm{Na}]^{+}$at $844.5 \mathrm{~m} / z$ elutes at $26.98 \mathrm{~min}$ (Fig. 2b). LC-MS/MS analysis of both strains showed that the MS/MS spectrum of $844 \cdot 5$ from strain DSM 4137 was identical to that from strain DSM 7348 (data not shown). To identify the meridamycin biosynthetic gene cluster, the recombinant plasmid construct pYH24 was introduced into DSM 4137 by conjugation using ET12657/pUZ8002 as donor host strain on SFM plates (Fig. 3). After incubation at $30^{\circ} \mathrm{C}$ for $18 \mathrm{~h}$, exconjugants were selected with $1 \mathrm{ml}$ water containing apramycin and nalidixic acid. Single colonies from this plate were transferred to an SFM plate containing apramycin for further confirmation of antibiotic resistance. Confirmed colonies were propagated on SFM plates without antibiotic selection to allow the double crossover to occur. To screen the potential double crossover mutants, single colonies from non-selective plates were replicated to an SFM plate containing $12.5 \mu \mathrm{g}$ apramycin $\mathrm{ml}^{-1}$. The candidates with the correct phenotype $\left(\mathrm{Apr}^{\mathrm{S}}\right)$ were chosen for further identification by PCR (Fig. 4a) and Southern blotting. Sequencing, hybridization (Fig. 4b) and subsequent LC-MS (Fig. 2c) results showed that disruption of the locus specifically abolished production of a metabolite with an LC-MS profile identical to that of authentic meridamycin from the known producer DSM 7348.

\section{Organization of the meridamycin biosynthetic gene cluster}

The meridamycin biosynthetic cluster is encoded by a $95 \mathrm{kbp}$ locus and encodes only five ORFs (Fig. 5, Table 1). The biosynthesis of the polyketide backbone of meridamycin is encoded by three large ORFs designated merA, merB and $m e r C$, which are adjacent and transcribed in the same direction and which encode, respectively, the multienzyme polypeptides MerA, MerB and MerC. Examination of the encoded AT domains of MER for distinctive amino acid sequence motifs allows the chemical nature of the extender unit recruited by each AT domain (especially, malonate versus methylmalonate or ethylmalonate) to be inferred (Haydock et al., 1995; Lau et al., 1999; Reeves et al., 2002; Del Vecchio et al., 2003). Examination of the 'reductive loop' (Staunton \& Weissman, 2001) domains [KR, DH, enoylreductsase (ER)] in each extension module allows the level of reduction to be deduced for that extension. In the case of hydroxyacyl extension units, even the stereochemical configuration of the hydroxy group can be predicted (Caffrey, 2003). The predicted polyketide product of the mer PKS from our analysis is entirely consistent with assembly of the polyketide chain of meridamycin, the modules being encoded along the chromosome in the order they are used from merA to merC (Table 2). Inspection of 


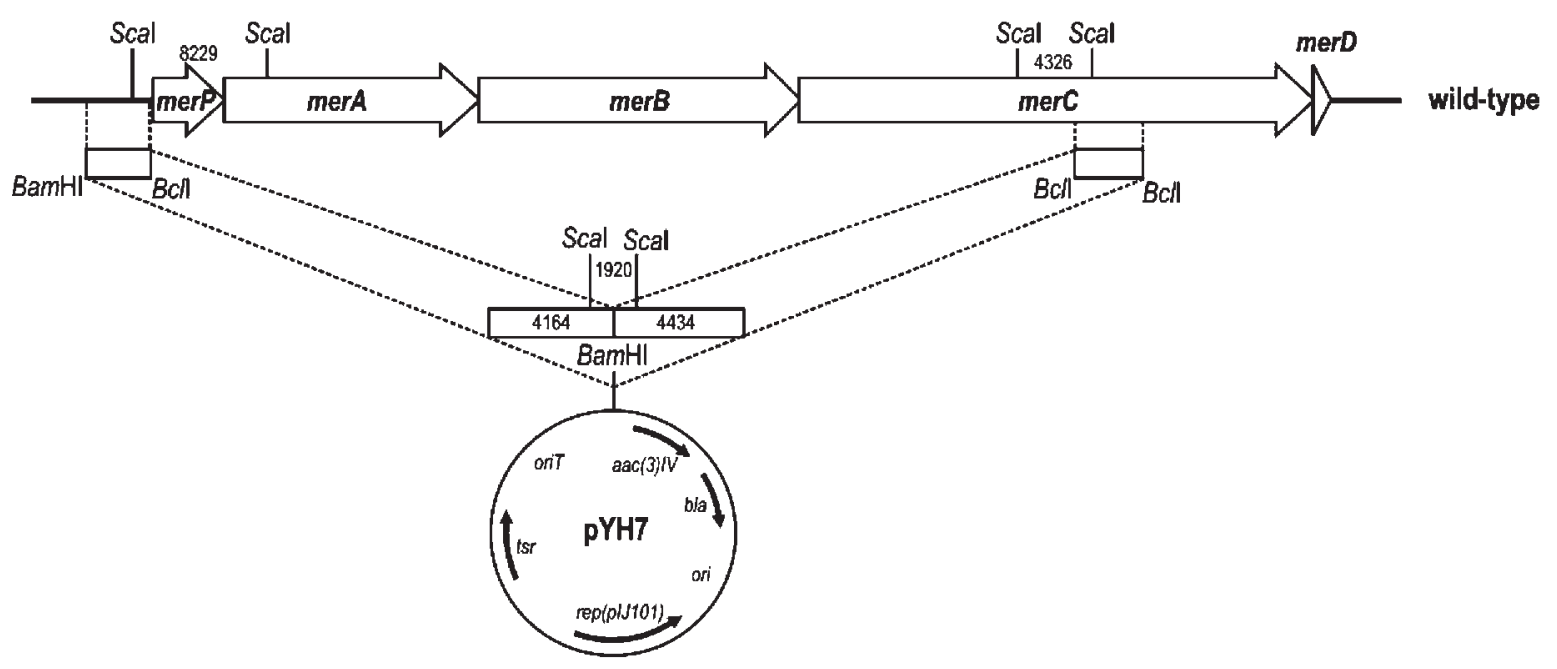

$\sqrt{ }$ Double crossover

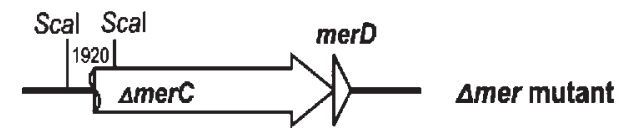

Fig. 3. Organization of the chromosomal mer genes before and after deletion. The numbers between Scal sites represent the expected size of the fragments hybridizing to the probe (1920 bp Scal fragment obtained from digestion of plasmid pYH24) after digestion of chromosomal DNA with Scal.

(a)

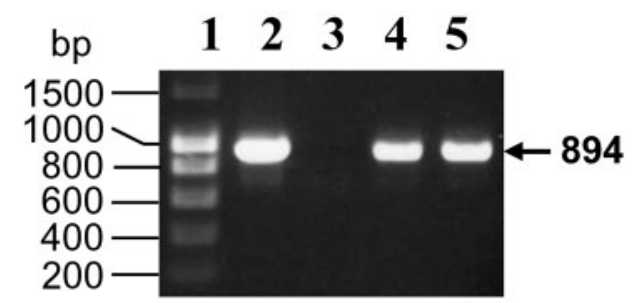

(b)

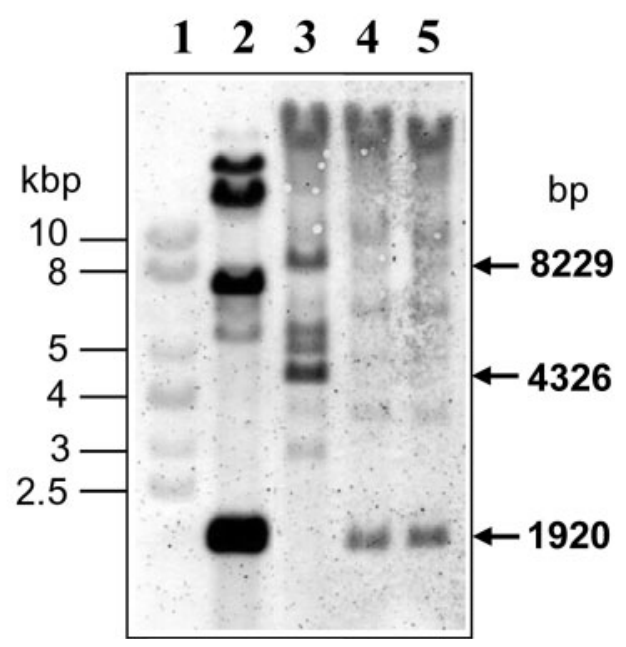

Fig. 4. Confirmation of gene disruption by PCR (a) and Southern blotting (b). A pair of primers, mer-3 and mer-4, covering the deleted region in the chromosome were used for quick screening to identify double crossover mutants. Southern blot analysis was performed with chromosomal DNA isolated from wild-type and mutants digested with Scal. The hybridization probe was the $1920 \mathrm{bp}$ Scal fragment obtained from digestion of plasmid pYH24, used for the mer cluster disruption. Plasmid pYH24 was digested with Scal and used as positive control. Extra bands hybridizing on the Southern blot are partial digestion products and cross-hybridization with other genomic fragments with PKS genes from the numerous other gene clusters in this organism. Lanes: 1, Hyper ladder I; 2, pYH24; 3, DSM 4137 (wild-type); 4, DSM 4137 $\Delta m e r$-a (mutant); 5, DSM $4137 \Delta$ mer-b (mutant). 


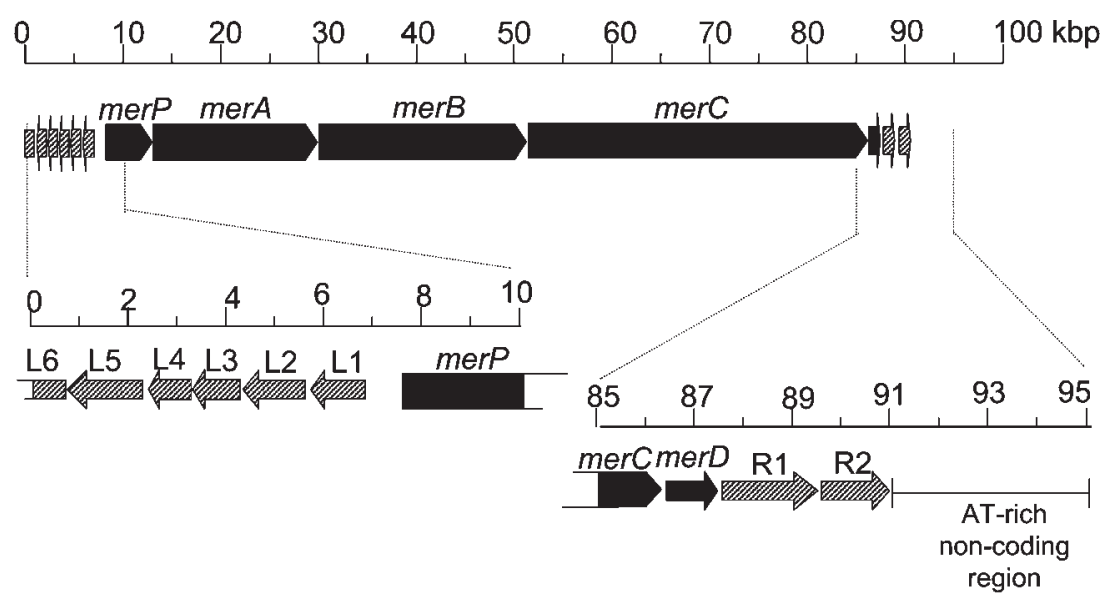

Fig. 5. Organization of the meridamycin biosynthetic locus in Streptomyces sp. DSM 4137. Genes proposed to be involved in meridamycin biosynthesis are coloured black. Hatched genes are not thought to be required for biosynthesis. the structure of meridamycin suggested that, if it is synthesized by a canonical modular PKS, the PKS can be predicted to comprise either a loading module for an acetate starter unit and 14 PKS extension modules, or a loading module that recruits a $\mathrm{C}_{5}$ starter unit followed by 13 extension modules. The analysis allows a clear choice in favour of the first of these possibilities: MerA (5721 aa, approx. $630 \mathrm{kDa}$ ) encodes the loading module (containing a KSQ-type decarboxylase) (Bisang et al., 1999) and extension modules 1-3; MerB (7178 aa, approx. $790 \mathrm{kDa}$ ) encodes extension modules 4-7; and MerC (11604 aa, approx. $1276 \mathrm{kDa})$ encodes modules for extensions $8-14$. The organization of the PKS for meridamycin shows close similarity to that of the related cluster from Streptomyces sp. NRRL 30748, with $95 \%$ identity at the protein level for most of the PKS translation.

There are two significant differences in the organization of the PKS genes in the two clusters. Module 14 in the mer cluster is unusual in that the expected ACP domain at the Cterminus of MerC is followed by a domain (about 400 aa) with significant end-to-end sequence similarity to a cytochrome P450 monooxygenase. Such an arrangement is to our knowledge unprecedented in modular PKS biosynthetic gene clusters that have been previously studied. This terminal P450 monooxygenase domain is absent from the counterpart PKS of strain NRRL 30748. It contains the conserved cysteine residue that provides a ligand to the haem iron in these enzymes. However, it lacks the absolutely conserved ExxR sequence upstream of the cysteine residue. The function of this domain is obscure, given that it is not present in the meridamycin PKS of NRRL 30748. Screening of the databases with this sequence identifies several putative cytochrome $\mathrm{P} 450$ monooxygenases, the best match being to a putative P450 from Nocardia farcinica IFM 10152, with $45 \%$ identity and $61 \%$ similarity at the protein level (accession no. BAD59368). This and other closely matching proteins also lack the ExxR sequence of the functional P450 haem oxygenase.

In Streptomyces sp. DSM 4137, MerC encodes extensions 8-14, whilst in Streptomyces sp. NRRL 30748 these

Table 1. Identifications of ORFs of the meridamycin biosynthetic gene cluster and flanking genes

\begin{tabular}{|lcll|}
\hline ORF & Amino acids & Best match, organism, (\% identity), (\% similarity) & Putative function \\
\hline L6 & Partial & ABC87052, Streptomyces NRRL 30748, (93), (96) & Mycodextranase \\
L5 & 532 & ABC87503, Streptomyces NRRL 30748, (93), (98) & $\alpha$-1,4-Glucosidase \\
L4 & 291 & ABC87504, Streptomyces NRRL 30748, (95), (98) & Sugar transporter \\
L3 & 318 & ABC87505, Streptomyces NRRL 30748, (97), (98) & Sugar transporter \\
L2 & 476 & ABC87506, Streptomyces NRRL 30748, (93), (96) & Sugar transporter \\
L1 & 336 & ABC87507, Streptomyces NRRL 30748, (95), (98) & LacI regulator \\
MerP & 1605 & ABC87508, Streptomyces NRRL 30748, (93), (95) & Non-ribosomal peptide synthetase \\
MerA & 5721 & ABC87509, Streptomyces NRRL 30748, (91), (93) & Polyketide synthase \\
MerB & 7178 & ABC87509, Streptomyces NRRL 30748, (91), (92) & Polyketide synthase \\
MerC & 11604 & ABC87509, Streptomyces NRRL 30748, (90), (92) & Polyketide synthase \\
MerD & 408 & BAD59369, Nocardia farcinica IFM 10152 (63), (76) & Cytochrome P450 monooxygenase \\
R1 & 687 & BAC70101, S. avermitilis MA-4680, (86), (88) & Amino acid permease \\
R2 & 445 & CAA16438, S. coelicolor A3(2), (79), (87) & RNA methyltransferase \\
\hline
\end{tabular}


Table 2. Organization of the meridamycin PKS

\begin{tabular}{|lll|}
\hline Mer protein & \multicolumn{1}{c|}{ Module } & \multicolumn{1}{c|}{ Domain structure } \\
\hline MerA & Loading & KSQ ATa ACP \\
& Extension 1 & KS ATp KR ACP \\
& Extension 2 & KS ATp DH KR ACP \\
& Extension 3 & KS ATa KR ACP \\
MerB & Extension 4 & KS ATe DH KR ACP \\
& Extension 5 & KS ATp KR ACP \\
& Extension 6 & KS ATp DH ER KR ACP \\
& Extension 7 & KS ATp DH KR ACP \\
MerC & Extension 8 & KS ATa KR ACP \\
& Extension 9 & KS ATa KR ACP \\
& Extension 10 & KS ATp KR ACP \\
& Extension 11 & KS ATa KR ACP \\
& Extension 12 & KS ATa KR ACP \\
& Extension 13 & KS ATp DH ER KR ACP \\
& Extension 14 & KS ATa ACP \\
& & \\
\hline
\end{tabular}

${ }^{\star} \mathrm{KS}$, ketosynthase; KSQ, decarboxylating ketosynthase; ATa, acyltransferase with malonyl specificity; ATp, acyltransferase with methylmalonyl specificity; ATe, acyltransferase with ethylmalonyl specificity; DH, dehydratase; ER, enoylreductase; KR, ketoreductase; ACP, acyl carrier protein.

extensions are reportedly split between two large ORFs designated MerC and MerD, in such a way that one module is split, with a C-terminal $\mathrm{KS}$ domain in $\mathrm{MerC}$ and an $\mathrm{N}$-terminal AT domain in MerD. Given this unusual arrangement and the otherwise very similar organization to that of the Streptomyces sp. DSM 4137 sequence, we examined this region more closely. The $\operatorname{mer} C$ and $\operatorname{mer} D$ genes occupy positions 64760-88666 and 88050-98327, respectively, of the deposited sequence from Streptomyces sp. NRRL 30748, that is, two genes are apparently coded for on the same strand and overlap by $600 \mathrm{bp}$. A more likely explanation, from comparison of the sequences, is that there is a single base error in the previously deposited sequence, so that a run of five guanines between positions 88303 and 88308 was incorrectly assigned as a run of six guanines. This correction would restore the reading frame and result in a contiguous sequence encoding a single ORF for extensions 8-14, just as in strain NRRL 4317.

Immediately upstream of merA lies another large ORF designated $\operatorname{merP}$ (1607 aa with an approximate molecular mass of $177 \mathrm{kDa}$ ), corresponding to merP in the deposited sequence from strain NRRL 30748, and encoding the protein MerP. MerP and its homologue in NRRL 30748 exhibit very high end-to-end sequence similarity to many authentic non-ribosomal peptide synthetase (NRPS) enzymes (comprising the domains in the following order: condensation, adenylation, peptidyl carrier protein and a further condensation domain). In particular, it has a high degree of identity to the NRPS proteins incorporating pipecolate, including the pipecolate-incorporating enzyme (PIE) of the biosynthetic gene clusters for other
FKBP-binding ligands such as rapamycin and FK506. MerP is therefore proposed to be responsible for addition of the pipecolate unit to the polyketide chain and subsequent formation of the macrocycle.

Immediately downstream of merC lies a single ORF predicted to encode a protein of 408 aa. This predicted gene product, MerD, shows end-to-end homology to authentic cytochrome P450 hydroxylases commonly encountered in macrolide gene clusters. In particular, it contains both the active-site cysteine and the conserved ExxR sequence. The meridamycin cluster in strain NRRL 30748 also has a P450 hydroxylase immediately downstream of the PKS. Interestingly, the genes from strains DSM 4137 and NRRL 30746 do not show particular homology and are no more alike than any two P450 hydroxylase genes. It also does not show particular homology to other P450 hydroxylases from either the rapamycin or FK506 gene clusters. The DSM 4137 sequence most resembles an authentic P450 gene from Nocardia farcinica IFM 10152 (accession no. BAD59369). It shows little similarity to the terminal domain of MerC. There is no position on the ring of meridamycin other than at C-9 that requires hydroxylation. Further work will be required to establish whether MerD, acting alone or in concert with the terminal domain of MerC, performs the oxidation at C-9 to give the characteristic 1,2,3-triketo arrangement in the FKBPbinding pharmacophore.

The probable limits of the mer gene cluster were probed by DNA sequence analysis. Upstream of merP is an apparently truncated version of an ornithine or lysine cyclodeaminase gene. This pseudogene would have been an obvious candidate to catalyse the synthesis of the pipecolate extender unit, if it had been intact. We have identified (S. F. H. and T. M., unpublished data) an intact copy of a cyclodeaminase gene in another gene cluster within this organism, adjacent to PKS genes. Possibly, the product of this gene is sufficient to provide pipecolate for both pathways. Immediately upstream of the presumed pseudogene are genes involved in carbohydrate metabolism. The first of these encodes a 532 aa protein with $93 \%$ identity to $\alpha$-1,4-glucosidase of Streptomyces sp. NRRL 30748 (accession no. ABC87503). This appears to be transcriptionally coupled to a gene encoding a protein with $94 \%$ identity to mycodextranase that also lies adjacent to the alpha-1,4-glucosidase above in the same organism (accession no. ABC87502). The organization of genes upstream of the PKS and NRPS components of the clusters in the two organisms is thus identical. The clearly identified function of these two genes appears to mark the upstream boundary of the cluster. To confirm this, we have sequenced a further $10 \mathrm{kbp}$ upstream and have identified no other genes that could be implicated in meridamycin biosynthesis. The downstream limits of the cluster were also explored by sequence analysis. Downstream of ORF1 is a gene encoding a putative 687 aa protein with $86 \%$ identity to a permease from Streptomyces avermitilis MA-4680. Adjacent to this is a gene 
predicted to encode a 445 aa protein with $79 \%$ identity to an RNA methyltransferase from Streptomyces coelicolor. Following this gene is a $5 \mathrm{kbp}$ region of higher AT content DNA that is not predicted to contain any ORFs. We have sequenced a further $10 \mathrm{kbp}$ beyond this and have again been unable to identify any genes that could be implicated in meridamycin biosynthesis. The different organization of genes downstream of ORF1 indicates that this gene marks the downstream limit of the gene cluster.

The mer cluster contains relatively few genes. In particular, there is an absence of regulation and export genes, and of genes for biosynthesis of the ethylmalonate extender unit. Such 'minimalist' clusters are a feature of Streptomyces sp. DSM 4137. Due to the extensive biosynthetic capacity, there appears to be some redundancy of function with genes shared between pathways. We have identified other functional clusters containing genes for ethylmalonate biosynthesis and pipecolate biosynthesis, and several that do not have associated export or regulatory genes (S. F. H. and T.M., unpublished data).

\section{Evidence for convergent evolution of the FKBP- binding pharmacophore}

Despite exhaustive screening for naturally occurring FKBPbinding macrocyclic polyketides, such metabolites are rare. Compared to the FK520, FK506 and rapamycin gene clusters, meridamycin shows some key differences in organization. Its final extension module encodes nonfunctional dehydratase and ketoreductase domains, rather than these being absent (Fig. 6), as well as bearing a pendant P450 domain. Also, although an ER domain is required for the complete reduction at $\mathrm{C}-12$ in all four compounds, the mer ER domain sequence is highly divergent from the equivalent domains of rapamycin, FK506 and FK520, which show very high mutual homology (data not shown). The biosynthesis of the FKBP-binding pharmacophore of meridamycin may have arisen by a process of convergent evolution to the other compounds.

\section{Concluding remarks}

The analysis of the meridamycin biosynthetic locus from Streptomyces sp. DSM 4137 has identified a set of modular PKS genes clustered with an NRPS-like gene and a gene for a cytochrome P450. The organization is similar to, but shows distinct differences from, the analogous gene cluster recently reported from Streptomyces sp. NRRL 30748. The minimal PKS cluster of DSM 4137 apparently recruits genes from other clusters within this organism to provide biosynthesis of the ethylmalonate and pipecolate extender units. The characterization of the genes for this interesting nonimmunosuppressant FKBP-binding macrolide, together with methods for the genetic manipulation of the producing strain, opens the way to genetic modification of the cluster to obtain novel structural variants of meridamycin with potentially improved bioactivity.

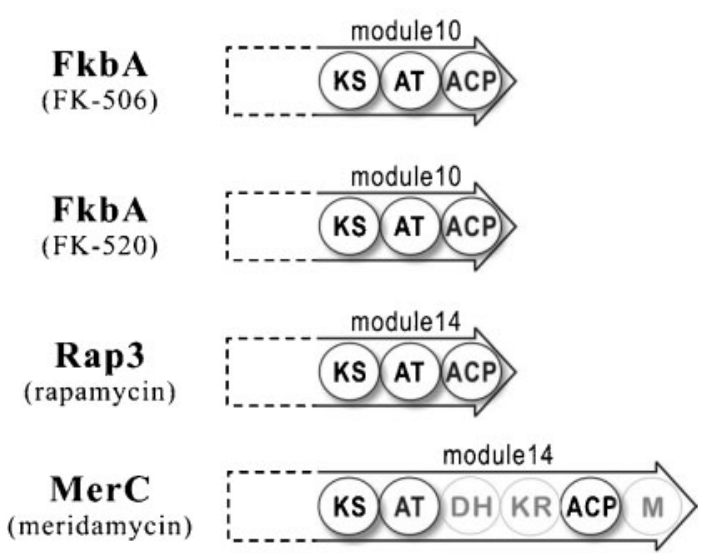

Fig. 6. Comparison among the last module in the FK506, FK520, rapamycin and meridamycin biosynthetic gene clusters. The arrows represent the PKS-encoded polypeptides. Domains within each module are represented by circles. Abbreviations: $\mathrm{KS}$, ketoacyl synthase; AT, acyltransferase; $\mathrm{DH}$, dehydratase; $\mathrm{KR}$, ketoreductase; ACP, acyl carrier protein; $\mathrm{M}$, cytochrome P450 monooxygenases.

\section{ACKNOWLEDGEMENTS}

This work was supported by the Wellcome Trust through an advanced Clinical Research Fellowship to S. F. H. and by the Royal Society China Fellowship to Y.S.

\section{REFERENCES}

Bisang, C., Long, P. F., Cortes, J. \& 7 other authors (1999). A chain initiation factor common to both modular and aromatic polyketide synthases. Nature 401, 502-505.

Bonfield, J. K., Smith, K. F. \& Staden, R. (1995). A new DNA sequence assembly program. Nucleic Acids Res 23, 4992-4999.

Caffrey, P. (2003). Conserved amino acid residues correlating with ketoreductase stereospecificity in modular polyketide synthases. Chembiochem 4, 654-657.

Del Vecchio, F., Petkovic, H., Kendrew, S. G. \& 7 other authors (2003). Active-site residue, domain and module swaps in modular polyketide synthases. J Ind Microbiol Biotechnol 30, 489-494.

Fehr, T., Sanglier, J.-J. \& Schuler, W. (1994). Rapamycin-like macrolide, and a new strain of streptomycete which produces it. International patent publication number WO 94/18207.

Gold, B. G., Densmore, V., Shou, W., Matzuk, M. M. \& Gordon, H. S. (1999). Immunophilin FK506-binding protein 52 (not FK506binding protein 12) mediates the neurotrophic action of FK506. $J$ Pharmacol Exp Ther 289, 1202-1210.

Gold, B. G., Armistead, D. M. \& Wang, M. S. (2005). Non-FK506binding protein-12 neuroimmunophilin ligands increase neurite elongation and accelerate nerve regeneration. J Neurosci Res 80, 56-65.

Guo, X., Dawson, V. L. \& Dawson, T. M. (2001). Neuroimmunophilin ligands exert neuroregeneration and neuroprotection in midbrain dopaminergic neurons. Eur J Neurosci 13, 1683-1693.

Hashimoto-Gotoh, T., Mizuno, T., Ogasahara, Y. \& Nakagawa, M. (1995). An oligonucleotide-directed dual amber method for sitedirected mutagenesis. Gene 23, 271-275. 
Haydock, S. F., Aparicio, J. F., Molnar, I. \& 7 other authors (1995). Divergent sequence motifs correlated with the substrate specificity of (methyl)malonyl-CoA:acyl carrier protein transacylase domains in modular polyketide synthases. FEBS Lett 374, 246-248.

Haydock, S. F., Mironenko, T., Ghoorahoo, H. I. \& Leadlay, P. F. (2004). The putative elaiophylin biosynthetic gene cluster in Streptomyces sp. DSM4137 is adjacent to genes encoding adenosylcobalamin-dependent methylmalonyl CoA mutase and to genes for synthesis of cobalamin. J Biotechnol 113, 55-68.

He, M., Halti, B., Summers, M., Feng, X. \& Hucul, J. (2006). Isolation and characterization of meridamycin biosynthetic gene cluster from Streptomyces sp. NRRL 30748. Gene 377, 109-118.

Kieser, T., Bibb, M. J., Buttner, M. J., Chater, K. F. \& Hopwood, D. A. (2000). Practical Streptomyces Genetics. Norwich: John Innes Foundation.

Lau, J., Fu, H., Cane, D. E. \& Khosla, C. (1999). Dissecting the role of acyltransferase domains of modular polyketide synthases in the choice and stereochemical fate of extender units. Biochemistry 38, 1643-1651.

Lubetski, C., Williams, A. \& Stankoff, B. (2005). Promoting repair in multiple sclerosis: problems and prospects. Curr Opin Neurol 18, 237-244.

MacNeil, D. J., Gewain, K. M., Ruby, C. L., Dezeny, G., Gibbons, P. H. \& MacNeil, T. (1992). Analysis of Streptomyces avermitilis genes required for avermectin biosynthesis utilizing a novel integration vector. Gene 111, 61-68.

Motamedi, H. \& Shafiee, A. (1998). The biosynthetic gene cluster for the macrolactone ring of the immunosuppressant FK506. Eur J Biochem 256, 528-534.

Paget, M. S. B., Chamberlin, L., Atrih, A., Foster, S. J. \& Buttner, M. J. (1999). Evidence that the extracytoplasmic function sigma factor $\sigma^{\mathrm{E}}$ is required for normal cell wall structure in Streptomyces coelicolor A3(2). J Bacteriol 181, 204-211.

Reeves, C. D., Chung, L. M., Liu, Y., Xue, Q., Carney, J. R., Revill, W. P. \& Katz, L. (2002). A new substrate specificity for acyl transferase domains of the ascomycin polyketide synthase in $S$. hygroscopicus. J Biol Chem 277, 9155-9160.
Salituro, G. M., Zink, D. L., Dahl, A., Nielson, J., Wu, E., Huang, L., Kastner, C. \& Dumont, F. J. (1995). Meridamycin: a novel nonimmunosuppressive FKB12 ligand from Streptomyces hygroscopicus. Tetrahedron Lett 36, 997-1000.

Sambrook, J., Fritsch, E. F. \& Maniatis, T. (1989). Molecular Cloning: a Laboratory Manual, 2nd edn. Cold Spring Harbor, NY: Cold Spring Harbor Laboratory.

Schwecke, T., Aparicio, J. F., Molnar, I. \& 10 other authors (1995). The biosynthetic gene cluster for the polyketide immunosuppressant rapamycin. Proc Natl Acad Sci U S A 92, 7839-7843.

Sosa, I., Reyes, O. \& Kuffler, D. P. (2005). Immunosuppressants: neuroprotection and promoting neurological recovery following peripheral nerve and spinal cord lesions. Exp Neurol 195, 7-15.

Staunton, J. \& Weissman, K. J. (2001). Polyketide synthases: a millennium review. Nat Prod Rep 18, 380-416.

Steiner, J. P., Connolly, M. A., Valentine, H. L., Hamilton, G. S., Dawson, T. M., Hester, L. \& Snyder, L. (1997). Neurotrophic actions of nonimmunosuppressive analogues of immunosuppressive drugs FK506, rapamycin and cyclosporin A. Nat Med 3, 421-428.

Sun, Y., Zhou, X., Liu, J., Bao, K., Zhang, G., Tu, G., Kieser, T. \& Deng, Z. (2002). 'Streptomyces nanchangensis', a producer of the insecticidal polyether antibiotic nanchangmycin and the antiparasitic macrolide meilingmycin, contains multiple polyketide gene clusters. Microbiology 148, 361-371.

Tanaka, K. \& Ogawa, N. (2004). Possibility of non-immunosuppressive immunophilin ligands as potential therapeutic agents for Parkinson's disease. Curr Pharm Des 10, 669-677.

Van Duyne, G. D., Standaert, R. F., Karplus, P. A., Schreiber, S. L. \& Clardy, J. (1991). Atomic structure of FKBP-FK506, an immunophilin-immunosuppressant complex. Science 252, 839-842.

Van Duyne, G. D., Standaert, R. F., Karplus, P. A., Schreiber, S. L. \& Clardy, J. (1993). Atomic structures of the human immunophilin FKB12 complexes with FK506 and rapamycin. J Mol Biol 229, 105-124.

Wu, K., Chung, L., Revill, W. P., Katz, L. \& Reeves, C. D. (2000). The FK520 gene cluster of Streptomyces hygroscopicus var. ascomyceticus (ATCC 14891) contains genes for biosynthesis of unusual polyketide extender units. Gene 251, 81-90. 